\title{
Untersuchung zum Ausfall der Strangführungsrollen in der horizontalen Zone einer Brammenstranggussanlage
}

\author{
Jörn Bräske*, Steffen Klohs, Thomas Mirre
}

\section{Zusammenfassung}

Es besteht ein Problem durch den häufigen Ausfall der Strangführungsrollen und die resultierenden erheblichen Verluste durch den Produktionsausfall, welcher beim Ersetzen der Rolleneinheiten entsteht.

Um die Ursachen zu ergründen wurden, zunächst die Randbedingungen und das Schadensbild analysiert. Daraus entstand die Hypothese, dass allgemein eine zu hohe Temperaturbelastung für die Schädigung der Lagerkomponenten verantwortlich ist und dies die grundlegende Ursache für die weitere Zerstörung darstellt.

Folglich musste eine Messeinrichtung installiert werden, um genauere Erkenntnisse über den Überhitzungsvorgang zu erlangen. Zu diesem Zweck wurden mehrere Verfahren zur Temperaturmessung miteinander verglichen, woraufhin eine Auswahl anhand einer Nutzwertanalyse erfolgte.

Die Auswertung der gewonnenen Messdaten zeigte, dass die Charakteristik der aufgezeichneten Überhitzungen sehr variabel ist. Weiterhin konnte keine Abhängigkeit von einem Prozessparameter ermittelt werden. Die genauer betrachteten Vorgänge konnten nicht oder nur eingeschränkt als Ursache für die Überhitzung nachgewiesen werden.

Wenngleich somit weitere Untersuchungen erforderlich sind, wurden mögliche Maßnahmen zur Lösung des Problems vorgeschlagen.

\section{Abstract}

The problem deals with the frequent malfunction of the guide rolls and the following serious loss through the lost output, which develops because of the replacement of the rolls.

First of all, the boundary conditions and the failure mode were analyzed to fathom the possible causes. Hence, the assumption arose that the general reason for the damage of the bearing components is that the temperature stress is too high, which is the reason for the further destruction. Consequently, a system of measurements has to be installed to get detailed insights about overheating. For this purpose, different methods of temperature detections were compared, whereupon a choice occurred on the basis of a utility analysis.

The evaluation of the achieved measurement data showed that the characteristic of the recorded overheating is very different. Furthermore, a dependence on a process parameter cannot be proved. It was not possible, or just restricted, to verify the eyed instances as a cause of the high temperature.

Although more researches are necessary, possible actions to solve the problem were proposed.

\section{Einführung in die Problemstellung}

Im Konverterstahlwerk der ArcelorMittal Eisenhüttenstadt $\mathrm{GmbH}$ wird aus flüssigem Roheisen zunächst die Stahlschmelze hergestellt, um aus ihr anschließend feste Halbzeuge zu fertigen. Letzteres geschieht in der Brammenstranggussanlage (Abb. 1 ), in deren Kokille, welche aus wassergekühlten Kupferplatten besteht, zunächst eine feste Strangschale entsteht. Der Strang verlässt die Kokille und wird durch die Kreisbogenanlage geführt, bevor er in der horizontalen Zone vollständig erstarrt. Daraufhin wird er per Querschnitt durch eine Brennschneidmaschine in Brammen geteilt, welche später im Warmwalzwerk weiterverarbeitet werden.

In der jüngeren Vergangenheit traten häufig Defekte an den Rolleneinheiten (Abb. 2) der horizontalen Strangführung auf. Diese bestehen aus drei einzeln auf einer fest stehenden Welle gelagerten Rollenkörpern. Zur Lage- rung dienen je ein Pendelrollen- und ein Torroidalrollenlager (CARB-Lager), welche durch eine Verbrauchsschmierung mit Schmierstoff versorgt werden. Zur Abdichtung der Lager dienen je ein Wellendichtring sowie ein Satz Doppellamellendichtringe. Zwischen den Rolleneinheiten sind seitlich Weitwurfdüsen angebracht, welche axial entlang der Rollenkörper Wasser spritzen, um diese zu kühlen. Die Drehbewegung der Rollenkörper wird, abgesehen von den Treiberrol- 

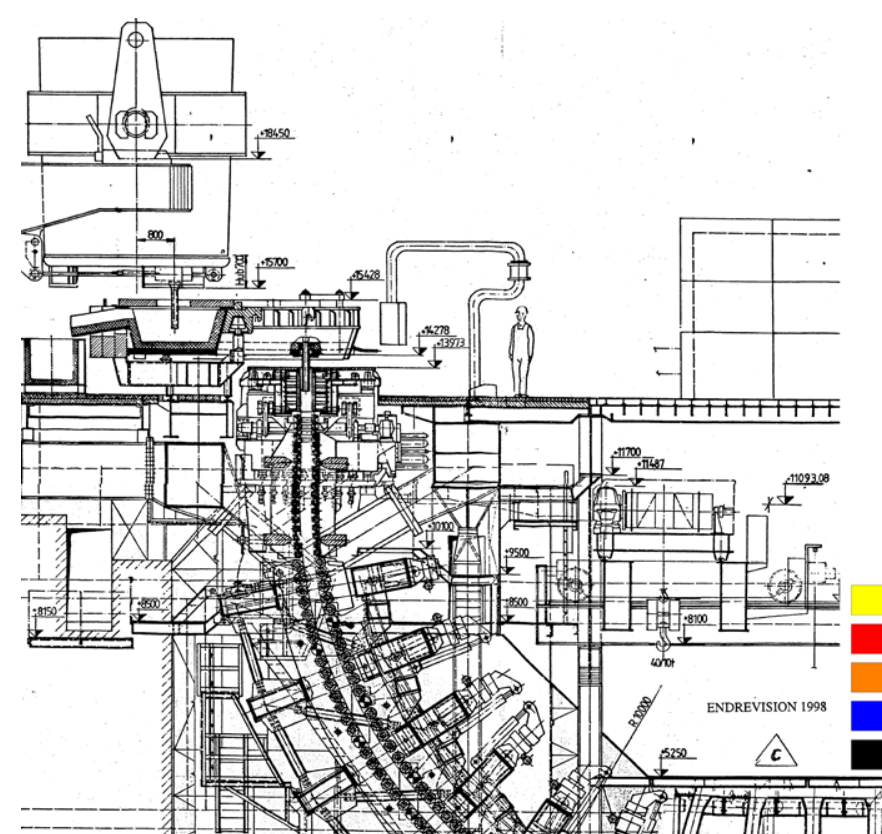

flüssiger Stahl erstarrter Stahl

Schlacke, Gießpulver

wassergekühlte Kupferplatten Feuerfest-Material, Rollen
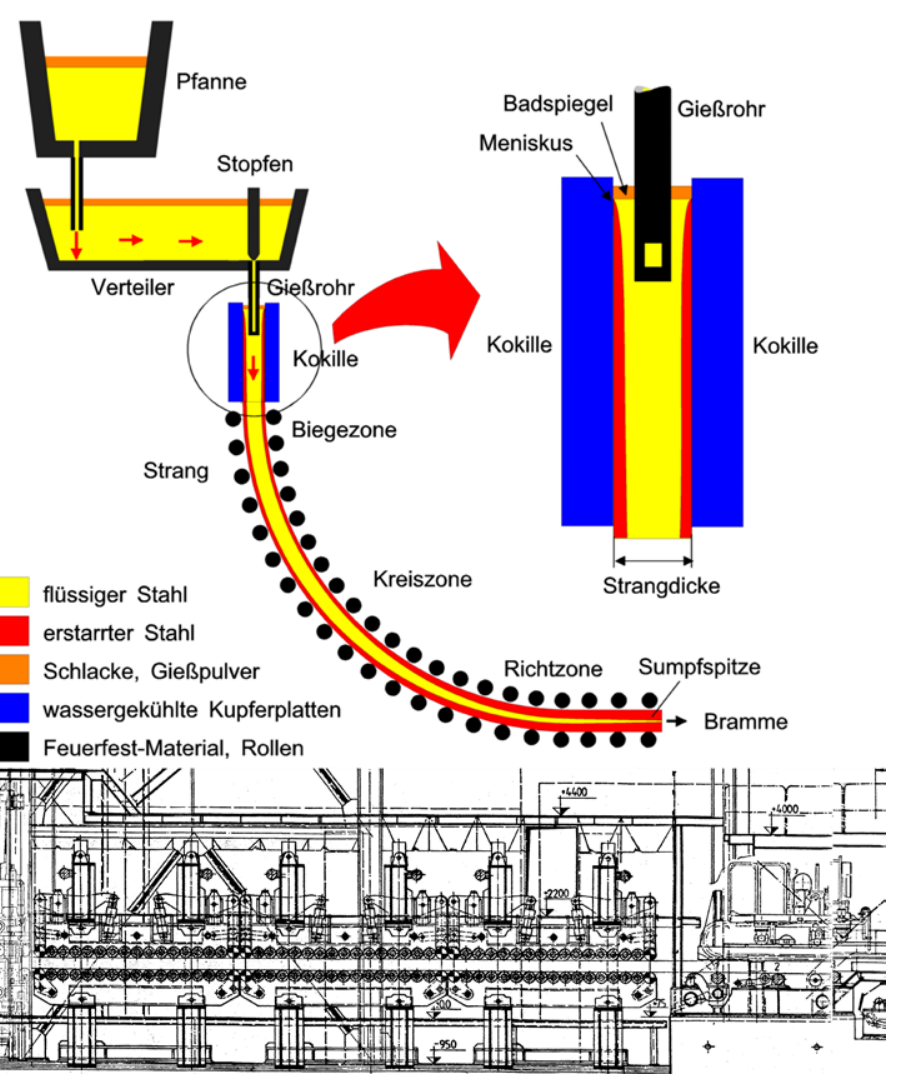

Abb. 1) Übersichtszeichnung und schematische Darstellung der Stranggussanlage

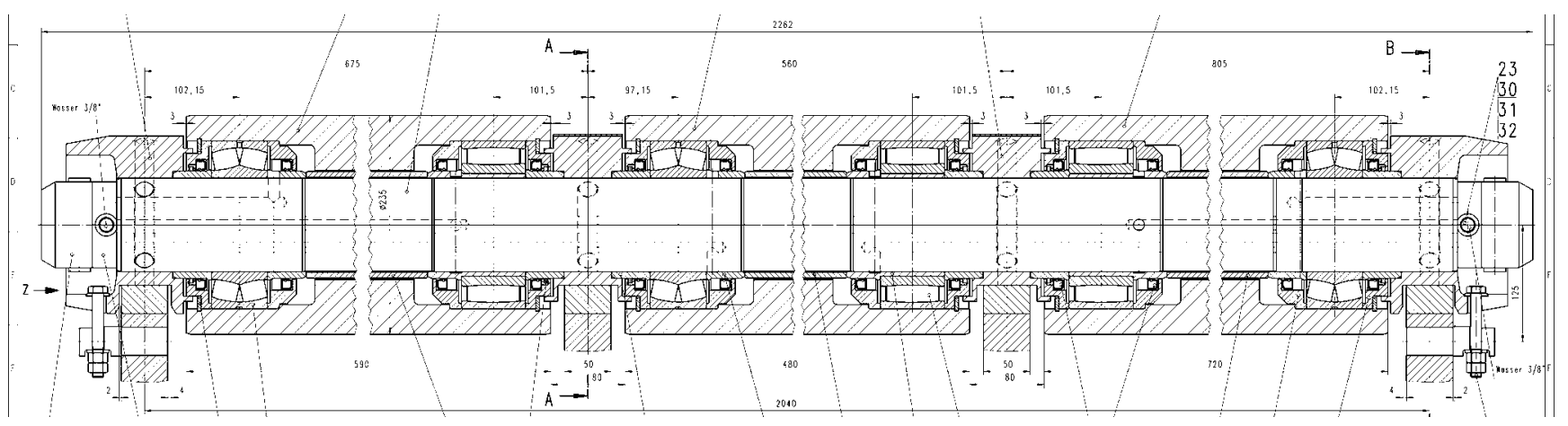

Abb. 2) Zeichnung Rolleneinheit

Ien, ausschließlich durch die Reibung am Strang hervorgerufen.

Die Defekte der Rolleneinheiten äußern sich auf unterschiedliche Art und Weise.

Wenn der Rollenkörper trotz Kontakt zum Strang nicht mehr rotiert, so entstehen Schleifspuren auf dem Rollenmantel sowie Riefen im Strang. Letztere stellen einen Qualitätsmangel dar, der sich meist auch auf die darauffolgenden Verarbeitungsprozesse auswirkt und diese negativ beeinflusst.

Ein weiteres Problem ist die Aufweitung des Gießspaltes, welcher durch den Abstand der gegenüberliegenden Rollenkörper definiert wird. Wenn dieser sich erweitert, so kommt es am
Strang, sofern dessen Kern noch flüssig ist, zu Ausbauchungen infolge des ferrostatischen Drucks.

Bei legiertem Stahl:

$$
\text { (1) } \begin{aligned}
\rho & =7900 \mathrm{~kg} / \mathrm{m}^{3} ; \\
\mathrm{h} & \approx 13 \mathrm{~m}(\text { ArcelorMittal } \mathrm{n} / \mathrm{a}) \\
p_{f} & =\rho \cdot g \cdot \mathrm{h} ; \\
p_{f} & =7900 \frac{\mathrm{kg}}{\mathrm{m}^{3}} \cdot 9,807 \frac{\mathrm{m}}{\mathrm{s}} \cdot 13 \mathrm{~m} \\
& =1007178,9 \frac{\mathrm{N}}{\mathrm{m}^{2}} \\
& =1007178,9 \mathrm{~Pa}
\end{aligned}
$$

wobei: $p_{f}$ : ferrostatischer Druck $[\mathrm{Pa}]$, $\rho:$ Dichte $\left[\mathrm{kg} / \mathrm{m}^{3}\right], \mathrm{h}$ : Höhendifferenz [m]
Die Folge dieser Querschnittserweiterung des Stranges können Mittenseigerungen sein, welche eine erhebliche Beeinträchtigung der Innenqualität darstellen (Schwerdtfeger 1992: 185). Weiterhin wiesen vereinzelte Wassersteinablagerungen auf dem Mantel der Rollen darauf hin, dass diese übermäßig erhitzt wurden. Diese Schlussfolgerung basiert auf der Tatsache, dass steinbildende Salze einen negativen Löslichkeitsindex haben und sich somit bei höheren Temperaturen stärker abscheiden. Auf eine lokale Überhitzung weisen auch die Anlassfarben auf der Manteloberfläche im Bereich der Lager hin. Weiterhin wurde beobachtet, dass der Schmierstoff nicht wie 
erwartet braun und pastös, sondern schwarz und dünnflüssig aus den Lagern austrat, was darauf hindeutet, dass zumindest dessen Tropfpunkt überschritten wurde.

Daraufhin wurden einzelne Rolleneinheiten untersucht. Vom Wellendichtring war meist nur noch der Federring aus Edelstahl vorhanden, an dem lediglich Rückstände des Fluorelastomers hafteten. Letzteres beginnt, laut Herstellerangaben, beim Überschreiten von $300^{\circ} \mathrm{C}$ sich dauerhaft chemisch zu zersetzen, wodurch keine Dichtwirkung mehr gegeben ist. Infolge dessen tritt Wasser in das Lager ein, was offensichtlich der Fall ist, wie an der Korrosion der Lagerbauteile zu erkennen ist. Der abrasive Verschleiß des Lagers wird dadurch enorm erhöht und es droht zu blockieren. Bei der Härteprüfung der Lager wurde dann auch die Wirkung der erhöhten Betriebstemperatur sichtbar. Die Lagerbauteile, insbesondere der Außenring, wiesen einen erheblichen Härteverlust von bis zu $35 \%$ auf. Dies ist auf die temperaturbedingten Gefügeveränderungen zurückzuführen, wodurch sich der Lagerverschleiß erhöht. Spätestens wenn durch eine defekte Rolleneinheit Qualitätsprobleme am Produkt auftreten, muss diese ausgetauscht werden. Dies ist nur bei einem Stillstand der Anlage möglich und dauert je nach Position der Rolle mehrere Stunden. Da die reguläre Rüstzeit zwischen den Sequenzen lediglich 35 Minuten beträgt, entstehen durch jeden Rollenwechsel hohe Produktionsausfälle, welche Verluste nach sich ziehen.

Die Variabilität der Schäden ließ auf verschiedene Schadensursachen oder zumindest unterschiedliche Abläufe des Schädigungsprozesses schließen. Allgemein wies das Schadensbild auf eine zu hohe Temperaturbelastung hin, wobei jedoch nur vage Vermutungen über deren Höhe und Einwirkdauer möglich waren.

Somit war ein Austausch einzelner Komponenten wenig sinnvoll, da die notwendige Temperaturbelastbarkeit, z. B. einer anderen Dichtung, nicht definiert werden konnte.

Dennoch wurde nach Produkten recherchiert, wobei jedoch keine zufriedenstellende Lösung gefunden wurde. Beispielsweise würde ein Schmierstoff

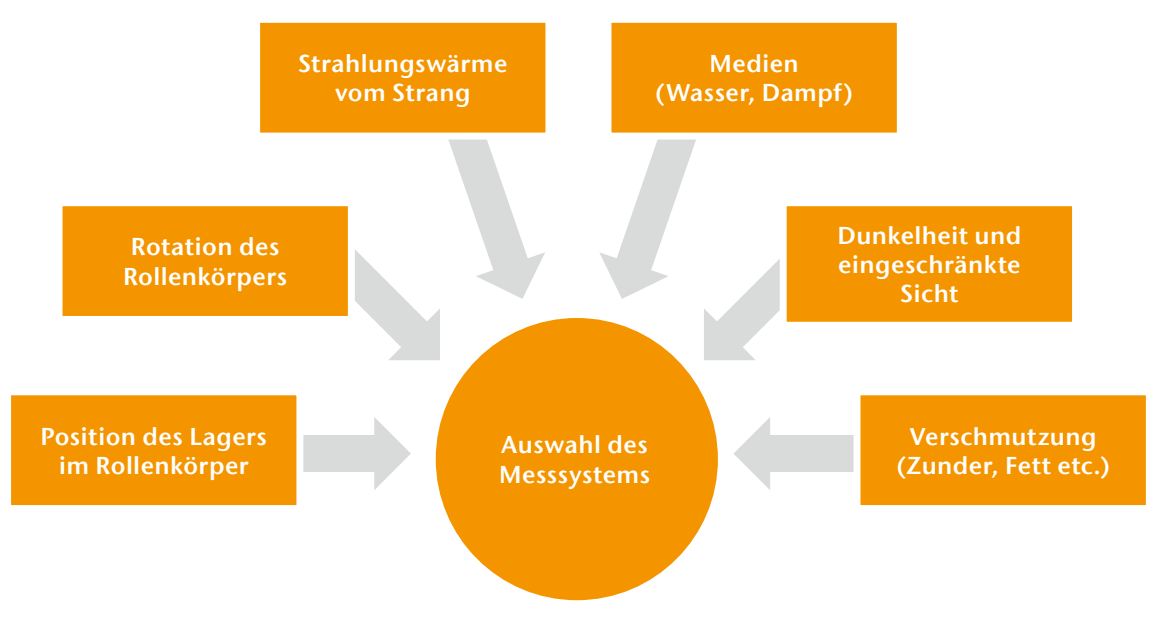

Abb. 3) Herausforderungen bei der Auswahl des Messsystems

mit einer Temperaturbelastbarkeit von bis zu $260{ }^{\circ} \mathrm{C}$ das 57 -Fache vom derzeit verwendeten kosten. Somit wäre diese Lösung, insbesondere bei der vorhandenen Verbrauchsschmierung, nicht wirtschaftlich.

Weiterhin wäre auch die Wirkung anderer kurzfristiger Maßnahmen, z.B. Erhöhung der Kühlung, nicht nachvollziehbar gewesen. Darüber hinaus sind bei der Veränderung der Kühlung stets die metallurgischen Einflüsse auf den Strang zu berücksichtigen.

Um die Ausfallursache genauer zu untersuchen und die Wirkung von Maßnahmen nachvollziehen zu können, war also die Einrichtung einer Temperaturmessung erforderlich.

\section{Vorbereitung, Durchführung und Auswertung der Untersuchungen}

\subsection{Auswahl und Einrichtung des Messsystems}

Die Anforderungen bei der Auswahl des Messsystems sind, wie Abb. 3 zeigt, ebenso hoch wie vielfältig. Nach der Recherche von vielfältigen Messverfahren wurden vier von ihnen genauer betrachtet und hinsichtlich ihrer Eignung untersucht. Zwei davon waren berührungslose, optische Messmethoden, welche anhand der elektromagnetischen Strahlung eines Körpers dessen Oberflächentemperatur bestimmen können: zum einen ein Pyrometer, welches die Temperatur an einem Punkt misst, und zum anderen eine Wärmebildkamera, mit der ein größerer Raum betrachtet werden kann. Die Temperatur des Lagerraums im Inneren des Rollenkörpers kann bei diesen beiden Verfahren also lediglich anhand der Temperatur des Rollenmantels abgeschätzt werden. Dennoch wäre somit eine Dauerüberwachung der Temperatur während der gesamten Sequenz möglich. Dagegen stellt eine andere Messmethode lediglich einen Maximalwertspeicher dar. Es handelt sich dabei um irreversible Temperaturindikatoren, die bei der Überschreitung einer bestimmten Temperatur einen Farbumschlag vollziehen. Sie sind sehr klein und können deshalb auch an schwer zugänglichen und sogar an bewegten Teilen angebracht werden. Somit ist die Anbringung direkt an den Lagerkomponenten möglich. Ihr Messbereich reicht jedoch, anders als bei Pyrometer und Thermovisionskamera, lediglich bis $224{ }^{\circ} \mathrm{C}$.

Weiterhin wäre zum Ablesen die Demontage der Rolleneinheit notwendig. Dies bedeutet, dass der Informationsgewinn erst nach dem Ausfall der Rolle erfolgen würde. Ein vorzeitiger Wechsel würde das Ziel, die Stillstandszeit zu minimieren, verfehlen.

Eine der häufigsten Methoden zur Temperaturmessung, insbesondere im Hochtemperaturbereich, ist ein Thermoelement. Dieses elektrische Messverfahren nutzt den Seebeck-Effekt bei Temperaturdifferenzen, um die dabei entstehende Potenzialdifferenz zu messen (Bernhard 2014: 832). Diese wird von der Auswertungseinheit auf die Temperaturskala abgebildet, sodass die Temperatur des Messkop- 
Tab. 1: Bewertung der ausgewählten Messverfahren

\begin{tabular}{|l|}
\hline Kriterium \\
\hline Erkenntnisgewinn \\
\hline Robustheit \\
\hline Aufwand \\
\hline Kosten \\
\hline Genauigkeit \\
\hline Summe \\
\hline Kriterium \\
\hline Erkenntnisgewinn \\
\hline Robustheit \\
\hline Aufwand \\
\hline Kosten \\
\hline Genauigkeit \\
\hline Summe \\
\hline
\end{tabular}

\begin{tabular}{l} 
Kriterium \\
Erkenntnisgewinn \\
Robustheit \\
\hline Aufwand \\
\hline Kosten \\
Genauigkeit \\
\hline Summe \\
\hline
\end{tabular}

\section{Kriterium}

Erkenntnisgewinn

Robustheit

Aufwand

Kosten

Genauigkeit

Summe

\section{Variante 1 Pyrometer \\ Punkte Begründung}

2 Dauermessung; nur punktuell

4 wassergekühltes Schutzgehäuse; Gefahr durch Kondensation

$4 \quad$ einfache Installation und Aufzeichnung

3 hohe Investition insb. bei Anschaffung mehrerer Geräte; Betriebskosten

3 geringer system. Fehler; Messung nur am Rollenmantel

16

\section{Variante 2 Thermografie}

Punkte Begründung

3 Dauermessung; Betrachtung peripherer Bauteile

3 luftgekühltes Schutzgehäuse, Gefahr durch Kondensation

$4 \quad$ einfache Installation und Aufzeichnung

3 hohe Investition insb. bei Anschaffung mehrerer Geräte; Betriebskosten

2 geringer systemat. Fehler bei guter Sicht; Messung nur am Rollenmantel

15

Variante 3

Temperaturindikator

Punkte

Begründung

3

lediglich Maximalwertspeicher; keine Abschätzung nötig, da direkte Messung

3 abgedichtet gegen Medien; mechanische Belastbarkeit und

Überhitzungsverhalten ungewiss

2

hoher Montageaufwand insbesondere bei Demontage zur Auswertung

5 sehr geringe Investitionskosten, keine Folgekosten während des Betriebs

3 sehr geringer Temperaturbereich; Messung direkt am Lager

16

Variante 4

Thermoelement

Punkte

4 Daueraufzeichnung; sehr gute Abschätzung der Lagertemperatur möglich

4 im biegsamen Mantel in feuerfestes Material eingebettet

3 einfache Installation und Aufzeichnung; Anpassung einzelner Bauteile notwendig

$4 \quad$ geringe Investitionskosten; sehr geringe Betriebskosten

4 sehr großer Temperaturbereich; geringer systemat. Fehler;

Messung sehr nah am Lager 


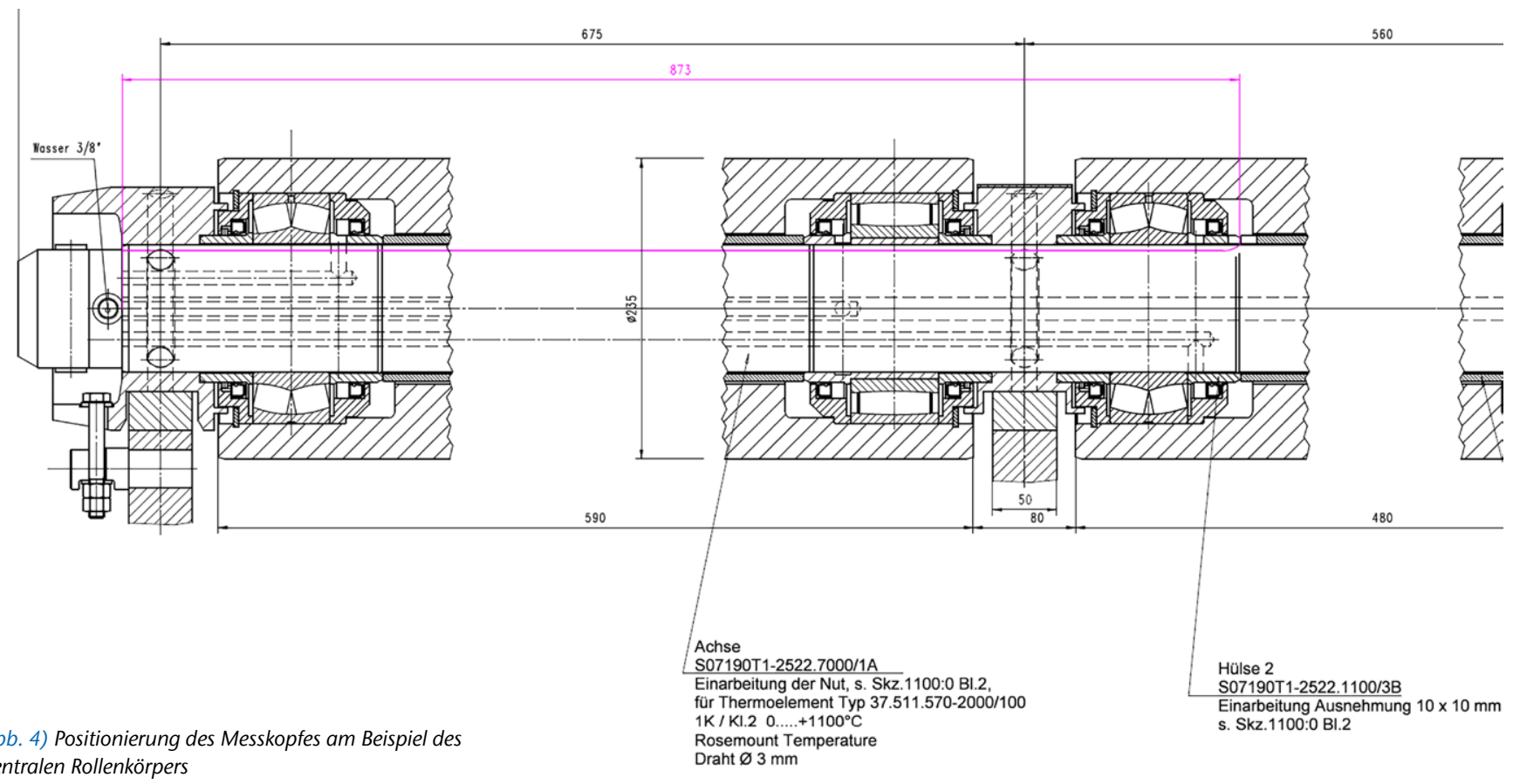

fes, an dem die beiden Thermodrähte miteinander verbunden sind, wiedergegeben wird. Für die vorliegende Aufgabe eignet sich die Bauform des Mantelthermoelementes, da diese zum einen biegsam und somit flexibel ist und zum anderen einen guten Wärmeübergang aufweist. Der Messkopf kann somit zumindest in der näheren Umgebung des Lagers positioniert werden, um eine möglichst repräsentative Dauermessung zu gewährleisten.

Für eine möglichst objektive Entscheidungsfindung wurden die verschiedenen Messmethoden in einer Nutzwertanalyse miteinander verglichen. Dazu wurden Kriterien definiert und entsprechend ihrer Priorität gewichtet. Anschließend erfolgte die Punktevergabe durch ein Bewertungssystem, welches sich an der VDI 2225 orientiert (Tab. 1). Das Ergebnis der Nutzwertanalyse wies das Mantelthermoelement als am besten geeignete Lösung aus (Tab. 2), sodass das Hauptaugenmerk bei der Untersuchung im Folgenden diesem Verfahren galt.

Der Messkopf des Thermoelementes sollte möglichst nah an das Lager geführt werden, die konkrete Ausführung geht aus Abb. 4 hervor. Da dieser Raum die gleiche radiale Position wie das Lager hat und ebenso von Rollenkörper, Abdrückring und Hülse begrenzt wird, kann von einem äquivalenten Wärmefluss ausgegangen werden. Die Temperaturüberwachung erfolgte jeweils am Pendelrollenlager des zentralen Rollen- körpers und am CARB-Lager des langen Rollenkörpers. Somit erfolgte die Messung bei verschiedenen Lagertypen und in verschiedenen Rollenkörpern, wodurch möglichst viele Erkenntnisse gewonnen werden sollten. In der Mitte der Rolleneinheit ist, aufgrund der höheren Temperatur des Strangzentrums und weil auch bei schmalen Gießbreiten Strangkontakt vorliegt, die höchste Temperaturbelastung zu erwarten. Deshalb wurde neben dem zentralen Rollenkörper der lange Rollenkörper gewählt, da dessen CARB-Lager zentraler positioniert ist als beim kürzeren, außen liegenden Rollenkörper.

Der Einbau der zur Temperaturmessung ausgestatteten Rollen sollte im Zuge der notwendigen Wechsel erfolgen, um zusätzliche Stillstandszeit zu vermeiden. Dennoch sollten die Positionen auch möglichst viele Erkenntnisse für die Untersuchungen liefern.

Folglich wurden die ersten 4 Rolleneinheiten je in den Innen- und Außenbogen der Rollen 4 und 8 im 3. Segment der horizontalen Strangführung eingebaut. Somit konnte zum einen untersucht werden, in welchem Maß die Temperaturbelastung zwischen den unten und oben liegenden Rollen differiert, und zum anderen sowohl Rollen unter den Querträgern als auch dazwischen kontrolliert werden. Die Rollenwechselstatistik wies nämlich,neben einem überwiegenden Ausfall im Innenbogen auch einen Schwerpunkt unter den Querträgern aus.
Einige Woche nach Beginn der Messung wurde außerdem die Rolle 5 im Innenbogen des 1 . Segments durch eine Temperaturmessrolle ersetzt, sodass auch die Betrachtung unterschiedlicher Segmente möglich war.

Wenngleich die ausgewählte Messmethode als am besten geeignet erschien, musste im Vorfeld betrachtet werden, in welchem Maß Abweichungen zwischen dem angezeigten Ergebnis und dem wahren Wert zu erwarten sind. Dabei mussten neben dem systematischen Fehler der Messeinrichtung auch zufällige Fehler, z. B. durch wechselnde Umwelteinflüsse sowie grobe Messfehler, z. B. durch falsche Montage, mit einbezogen werden. Für die Auswertung und Interpretation der Messergebnisse ist, bei grundlegend funktionierendem Messaufbau, insbesondere die Angabe der Grenzabweichung von Bedeutung. Sie wird vom Hersteller angegeben und beträgt bei Klasse 2:

\section{$2 \pm 2,5{ }^{\circ} \mathrm{C}$ oder $\pm 0,0075 \cdot \mathrm{T}\left({ }^{\circ} \mathrm{C}\right)$ $\mathrm{T}$ : Temperatur (Rössel-Messtechnik 2012: 3)}

Dabei ist zu beachten, dass jeweils der größere Wert gilt.

Zur Visualisierung der Messdaten diente das Programm ibaAnalyzer, mit dem ausgewählte Prozessdaten vom Server abgerufen werden können. Somit konnten neben den Temperaturwerten auch zur Auswertung relevante 
Tab. 2: Ergebnis der Nutzwertanalyse

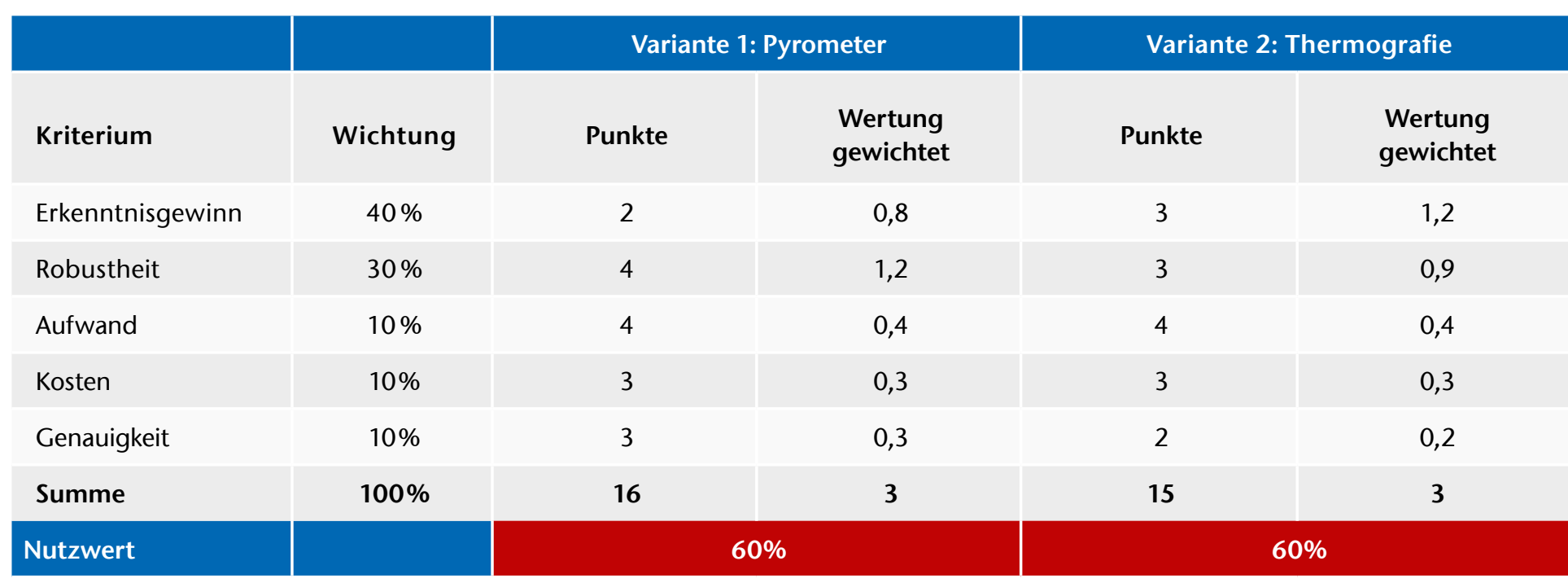

Daten, wie z. B. die Gießgeschwindigkeit, abgerufen werden.

\subsection{Analyse der Messdaten und} weiterführende Betrachtungen

Nach Betrachtung des Temperaturverlaufs über mehrere Sequenzen war es möglich, den regulären Betriebszustand zu beschreiben. So wurde festgestellt, dass sich die Betriebs- temperatur während der Sequenz, bei geringfügigen Schwankungen, in folgendem Bereich befindet:

$$
\text { (3) } \begin{aligned}
& \mathrm{T}_{\text {Betrieb }} \approx 80^{\circ} \mathrm{C} \pm 2,5^{\circ} \mathrm{C} \text { bis } \\
& 100{ }^{\circ} \mathrm{C} \pm 2,5^{\circ} \mathrm{C}
\end{aligned}
$$

Um festzulegen, wann von einer Überhitzung gesprochen werden kann, erfolgte die Orientierung an der Temperaturbelastbarkeit der Bauteile. Die Schwachstelle stellte dabei der Wellendichtring dar, welcher bei Temperaturen über $200^{\circ} \mathrm{C}$ weich wird, wodurch sich seine Anpresskraft und langfristig auch die Verschleißfestigkeit verringern. Der Überhitzungszustand wurde somit durch Temperaturen über $200{ }^{\circ} \mathrm{C}$ definiert.

Die erste Auffälligkeit war jedoch keine Überhitzung, sondern die Tatsache, dass der Temperaturbereich bei Stahlmarken für Elektroanwendun-
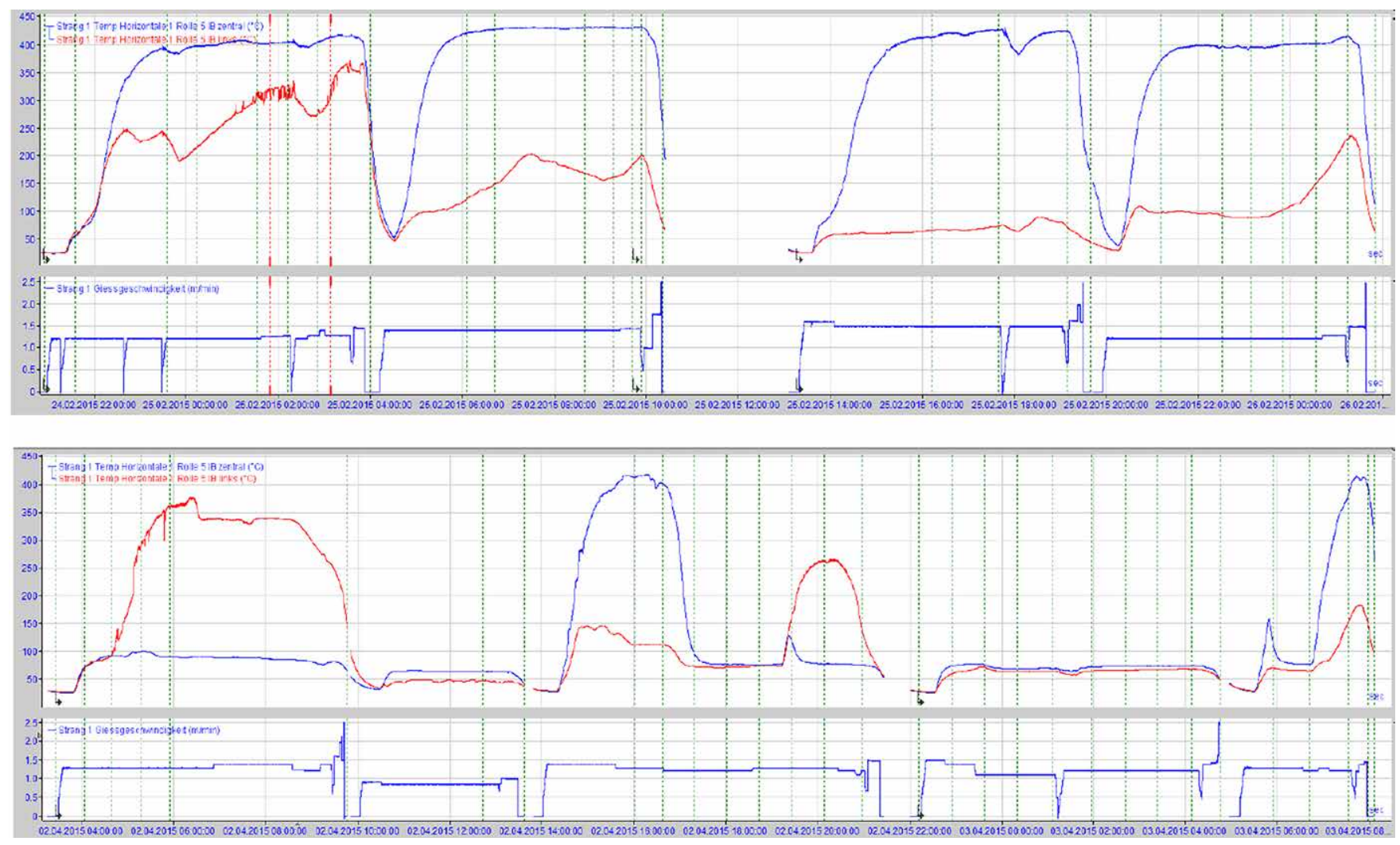


\begin{tabular}{|c|c|c|c|c|c|}
\hline Punkte & $\begin{array}{c}\text { Wertung } \\
\text { gewichtet }\end{array}$ & Punkte & $\begin{array}{c}\text { Wertung } \\
\text { gewichtet }\end{array}$ & Punkte & $\begin{array}{c}\text { Wertung } \\
\text { gewichtet }\end{array}$ \\
\hline 3 & 1,2 & 4 & 1,6 & 5 & 2 \\
\hline 3 & 0,9 & 4 & 1,2 & 5 & 1,5 \\
\hline 2 & 0,2 & 3 & 0,3 & 5 & 0,5 \\
\hline $\mathbf{3}$ & 0,5 & 4 & 0,4 & 5 & 0,5 \\
\hline $\mathbf{1 6}$ & 0,3 & 4 & 0,4 & 5 & $\mathbf{2}$ \\
\hline
\end{tabular}

gen deutlich geringer ist als bei allen anderen Güten:

$$
\text { (4) } \begin{aligned}
\mathrm{T}_{\text {Elektrostahl }} \approx & 60^{\circ} \mathrm{C} \pm 2,5^{\circ} \mathrm{C} \text { bis } \\
& 80^{\circ} \mathrm{C} \pm 2,5^{\circ} \mathrm{C}
\end{aligned}
$$

Als Ursache ist vorrangig die stärkere Wärmeabfuhr aus der Strangschale durch erhöhte Kühlwassermengen und geringere Gießgeschwindigkeit anzuführen. Notwendig ist dies, um die Durcherstarrung des Stranges trotz einer geringeren Wärmeleitfähigkeit, aufgrund des erhöhten Siliziumgehalts, zu gewährleisten.

Die Charakteristik der detektierten Überhitzungen war sehr variabel, wie Abb. 5 beispielhaft veranschaulichen soll. Zunächst erkennt man hierbei den voneinander unabhängigen Temperaturverlauf der beiden benachbarten Rollenkörper. So weist der zentrale Rollenkörper (blauer Graph) in den oben dargestellten Sequenzen jeweils eine starke Überhitzung über die gesamte Dauer der Sequenz auf, während der linke Rollenkörper (roter Graph) nur zwischenzeitlich oder nicht überhitzt. Weiterhin ist die Intensität der Überhitzungsereignisse hinsichtlich Höchsttemperatur und Einwirkdauer sehr unterschiedlich.

\section{(5) zentraler Rollenkörper:} $\mathrm{T}_{\max } \approx 425^{\circ} \mathrm{C} \pm 3,2{ }^{\circ} \mathrm{C}$

6 linker Rollenkörper: $\mathrm{T}_{\max } \approx 365^{\circ} \mathrm{C} \pm 2,7^{\circ} \mathrm{C}$
Somit kann auch davon ausgegangen werden, dass der Schädigungsverlauf der Lagerkomponenten variabel ist.

Die Temperaturschwankungen konnten nicht auf ein Gießereignis (z. B. Stranghalt) zurückgeführt werden. Deshalb wurden die Überhitzungsereignisse dokumentiert und hinsichtlich ihrer Abhängigkeit von verschiedenen Prozessparametern ausgewertet. In dieser tabellarischen Dokumentation wurden u. a. die während der Überhitzungen vergossenen Stahlmarken notiert, da diese Unterschiede bezüglich der chemischen Zusammensetzung aufweisen. Damit verbunden sind auch variable Eigenschaften z.B. bezüglich des Erstarrungsverhaltens. Weiterhin wurde die Gießgeschwindigkeit vermerkt, da auch diese die Erstarrung und die damit verbundenen Ausbauchungen des Stranges beeinflusst. Außerdem wurde dokumentiert, in welchem Rahmen sich die Gießbreite bewegte. Diese hat aufgrund der von ihr bestimmten Kontaktfläche zwischen dem Strang und den äußeren Rollen Einfluss auf deren Wärmeeintrag und Antrieb. Darüber hinaus wurde auch die angewandte Kühlgruppe vermerkt, da diese großen Einfluss auf die Wärmeabfuhr vom Strang und von den Baugruppen hat. Um zu untersuchen, ob die Überhitzung von der vergossenen Stahlmarke abhängig ist, wurden die notierten Stahlmarken entsprechend ihrer Häufigkeit in einem Säulendiagramm dargestellt (Abb. 6a). Die Abhängigkeit der Ereignisse von einer Stahlmarke konnte dabei jedoch nicht festgestellt werden. Selbst die Stahlmarke, welche am häufigsten in Sequenzen mit Überhitzungen vergossen wurde, stellt lediglich einen Anteil von 22,8 \% an den Gesamtereignissen dar.

Weiterhin wurde die Intensität der Kühlung betrachtet, wobei zu beachten ist, dass die Stärke der Kühlwirkung von Gruppe 1 zu Gruppe 5 hin ansteigt (Abb. 6b).

Kühlgruppe 6 ist ein Sonderfall, da die Kühlung hier wiederum relativ weich erfolgt.

Da jedoch kein Trend erkennbar war, kann weder eine starke noch eine schwache Kühlung als Ursache für die Überhitzungen festgemacht werden.

Weiterhin wurde der Mittelwert von Gießgeschwindigkeit und -breite während der Sequenzen, in denen es zu Überhitzungen kam, gruppiert und ausgewertet (Abb. 7).

Auch bei diesen Parametern ließ sich kein Trend zu einem der Randbereiche nachweisen. Vielmehr deuten die Diagramme auf eine Normalverteilung der Ereignisse hin, wobei für solche statistischen Bewertungen eine mengenneutrale Auswertung notwendig wäre.

Parallel zur Temperaturmessung wurden weitere Untersuchungen durchgeführt, um Erkenntnisse zur Ausfallursache der Strangführungsrollen zu erlangen. So sollte, als die Überhitzungen häufiger festgestellt wurden, der Zusammenhang zwischen Rotation und Überhitzung überprüft werden. Die Grundlage hierfür war, dass die Weitwurfdüsen nicht den kompletten 


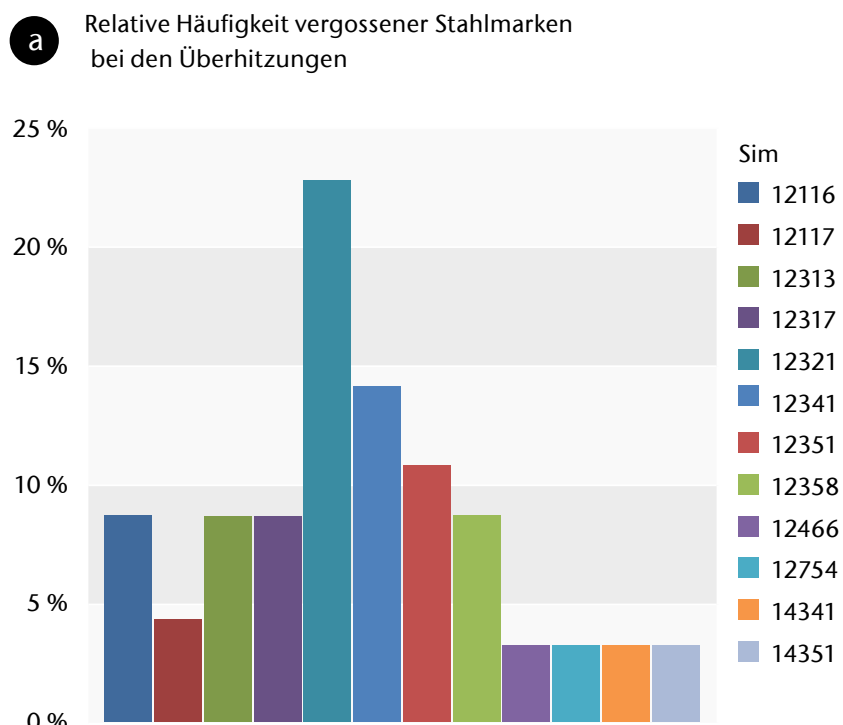

Abb. 6) Abhängigkeit der Überhitzungen von der Stahlmarke (a) und Kühlgruppe (b) b Relative Häufigkeit angewandter Kühlgruppen bei den Überhitzungsereignissen

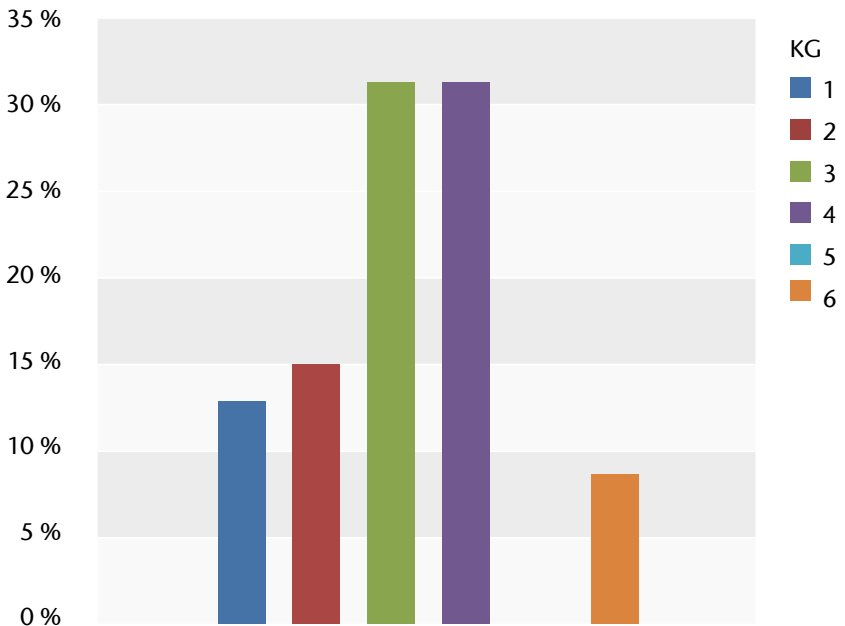

a Relative Häufigkeit der gefahrenen mittleren Gießgeschwindigkeit bei den Überhitzungen

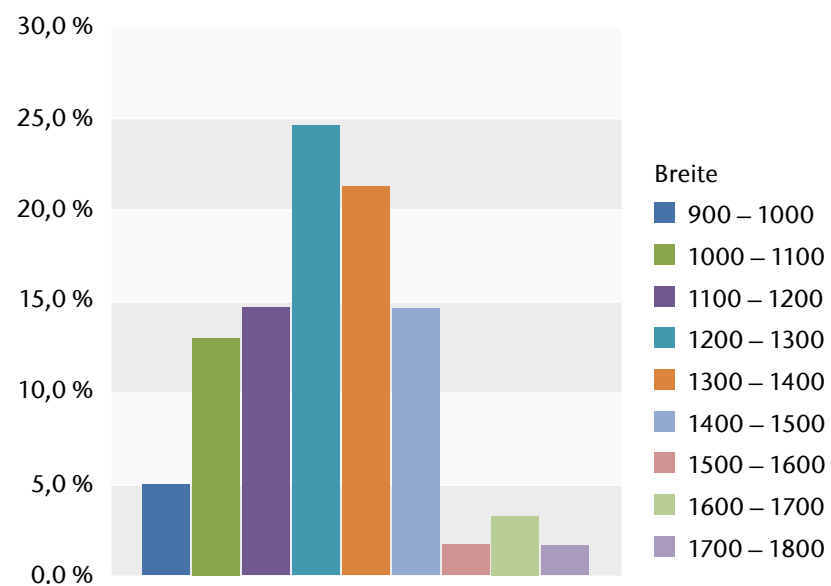

(b)

Relative Häufigkeit der mittleren eingestellten Gießbreite bei den Überhitzungen

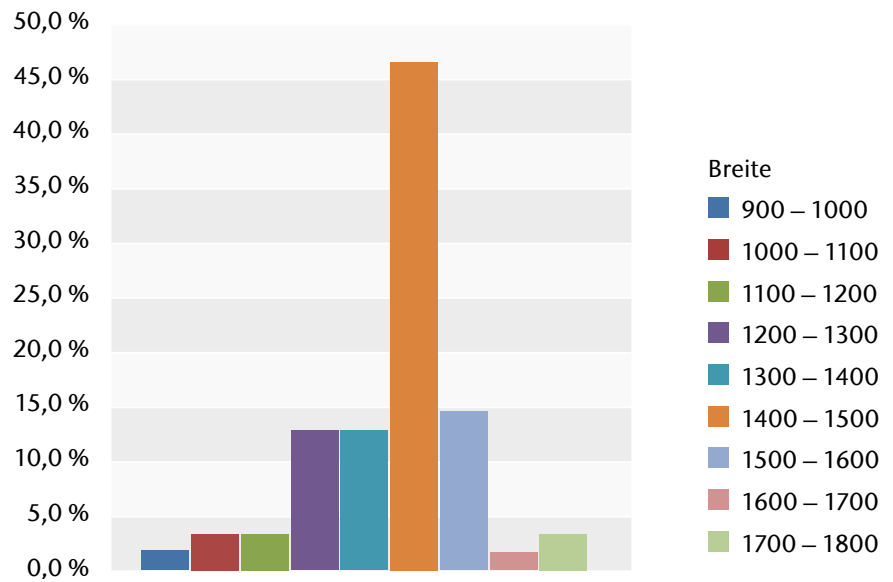

Abb. 7) Abhängigkeit der Überhitzungen von der Gießgeschwindigkeit (a) und-breite (b)

Umfang des Rollenmantels kühlen, wenn dieser nicht mehr rotiert.

$\mathrm{Zu}$ diesem Zweck wurde ein Rollenkörper, der mit einem Thermoelement ausgestattet war, per Videokamera überwacht. Diese wurde durch ein Kühlgehäuse wassergekühlt und ein Luftblasvorsatz sollte eine gute Sicht auf die Rollenoberfläche gewährleisten. Der einzigen Überhitzung der überwachten Rolle während der Kameraaufzeichnung ging deren Stillstand voraus. Da die Uhr-Anzeige der Software defekt war, konnte der Zeitpunkt nicht genau zugeordnet, sondern nur anhand der Aufzeichnungsdauer abgeschätzt werden. Der Nachweis des Zusammenhanges war somit nur eingeschränkt und nicht wie gewünscht repräsentativ möglich. Weiterhin sollte untersucht werden, ob innerhalb des Rollenteppichs der horizontalen Strangführung lokale Schwerpunkte der Überhitzung festgestellt werden können. Zu diesem Zweck ist die installierte Messmethode nicht geeignet, da es mit einem sehr großen Aufwand verbunden wäre, alle Rollen mit Thermoelementen auszustatten. Deshalb wurden die Rollen, unmittelbar nachdem der Strang zum Sequenzende die Kühlkammer verlassen hat, mit Hilfe einer Thermografiekamera begutachtet. Die erste Erkenntnis war, dass die lokale Temperaturverteilung nicht nur innerhalb des Rollenteppichs, sondern auch bei der Betrachtung eines überhitzten Rol- lenkörpers sehr unterschiedlich sein kann (Abb. 8).

Aufgrund des geringen Stichprobenumfangs konnte keine repräsentative statistische Auswertung erfolgen. Dennoch ist die Information, dass bei $82 \%$ der Überhitzungen an allen 3 Segmenten die Rollen 12-15 betroffen waren, eine interessante Grundlage für weitere Untersuchungen und mögliche Maßnahmen. Die Tatsache, dass im Außenbogen keine Überhitzung festgestellt wurde, bestätigt die bisherige Annahme aus der Temperaturmessung durch die Thermoelemente, dass von der Temperaturproblematik nur der Innenbogen betroffen ist.

Auch das Schadensbild der defekten Rolleneinheiten wurde bei deren De- 
montage begutachtet. Dabei wurden zunächst Riefen am Verschlussring (Abb. 9a) sowie am Innenlagerbock (Abb. 9b) festgestellt, was auf starke Reibung zwischen diesen beiden Komponenten schließen lässt. Außerdem war der Sicherungsring gebrochen, sodass eine Sicherung der axialen Position nicht mehr gegeben war. Auch der einseitig starke Verschleiß des CARB-Wälzkörpers, insbesondere an dessen Stirnseite, lässt auf falsche axiale Positionierung schließen (Abb. 9d). Die axiale Verschiebung des Loslagers wird zuerst durch den 3-mmSpalt zwischen Rollenkörper und Lagerbock begrenzt, wobei dieser oft durch feste Ablagerungen vermindert wird (Abb. 9c).

Darüber hinaus wurde bei ausgebauten, aber noch montierten Rolleneinheiten eine Unwucht festgestellt, die auf eine übermäßige Biegung der Achse schließen lässt.

Daraufhin wurde untersucht, ob die radiale Lagerluft des CARB-Lagers durch Schiefstellung infolge der Biegung und durch axiale Verschiebung so stark gemindert werden kann, dass das Lager blockiert. Die Berechnungen ergaben jedoch, dass ein Blockieren des Lagers theoretisch nicht als Ursache für eine Überhitzung in Frage kommt, auch dann nicht, wenn sich das ursprüngliche Lagerspiel an der unteren Grenze des Toleranzfeldes (185 $\mu \mathrm{m})$ befand. Andersherum kann es im Überhitzungsfall durchaus zum Blockieren des Lagers kommen, da die radiale Lagerluft sich dann verringert und nur noch eine axiale Verschiebung von 2,32 mm zulässt.

\section{Zusammenfassung und Ausblick}

Kernpunkt der Untersuchungen war die Temperaturmessung mittels Thermoelementen im Inneren der Rolleneinheiten. Diese lieferte eine Vielzahl von Informationen, insbesondere bezüglich des zeitlichen Verlaufs. So konnten neben dem regulären Betriebszustand auch Effekte infolge von Gießereignissen festgestellt werden. Weiterhin wurde das Maß der lokalen Temperaturdifferenzen zwischen benachbarten und gegenüberliegenden Rollenkörpern ermittelt. Das Hauptaugenmerk lag jedoch auf der Analyse der Überhitzungsereignisse. Diese

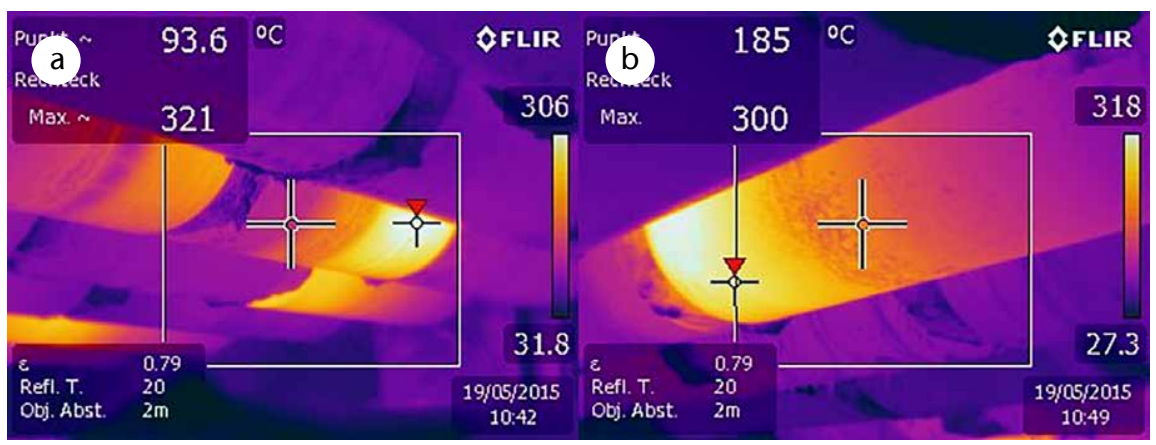

Abb. 8) Lokale Temperaturverteilung an den Strangführungsrollen (Thermografiekamera)

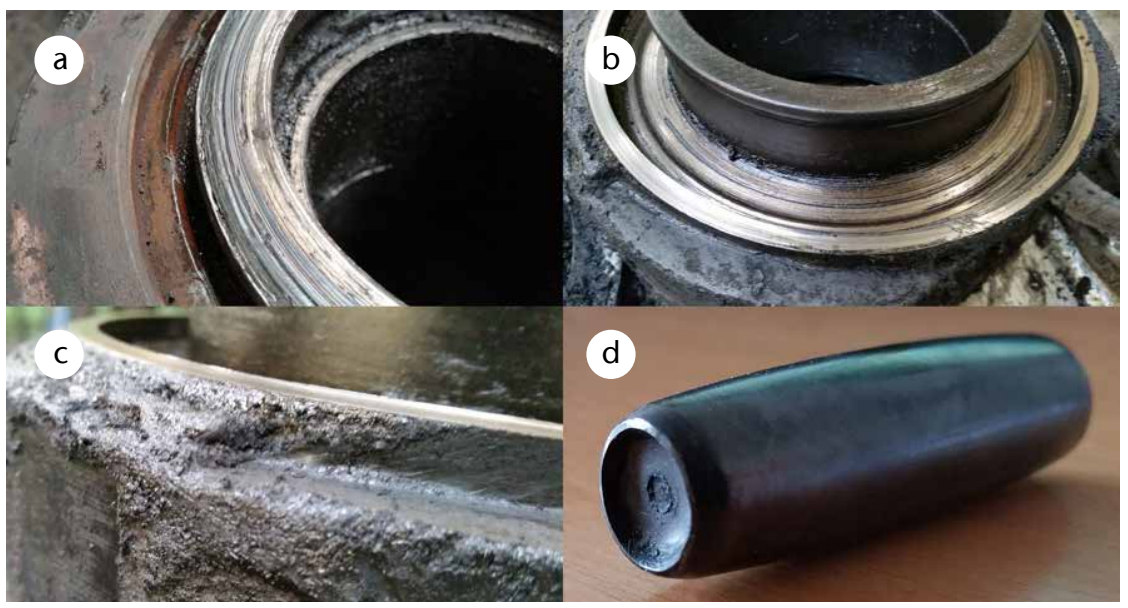

Abb. 9) Zusammengefasste Schadensbilder bei der Demontage der Rolleneinheit

wiesen eine große Variabilität hinsichtlich der gemessenen Temperatur, des Zeitpunktes und der Dauer des Auftretens sowie ihres zeitlichen Verlaufs auf. Auch deshalb konnte bei Betrachtung der Prozessdaten kein Gießereignis als Ursache für die Überhitzungen festgestellt werden. Bei der Auswertung der dokumentierten Ereignisse hinsichtlich ihrer Abhängigkeit von der vergossenen Stahlmarke, der angewandten Kühlgruppe sowie der gefahrenen Gießgeschwindigkeit und -breite war kein Trend erkennbar. Der Zusammenhang zwischen der Rotation eines Rollenkörpers und dessen Überhitzung konnte nur eingeschränkt nachgewiesen werden. Die Untersuchung des Rollenteppichs in der horizontalen Strangführung mit Hilfe einer Thermografiekamera bestätigte, dass sich die Überhitzungen auf den Innenbogen beschränken.

Eine erneute Begutachtung des Schadensbildes der Rolleneinheit wies auf ein Problem hinsichtlich der axialen Verschiebung hin. Als Engpass wurde der 3-mm-Spalt zwischen Rollenkörper und Mittenlagerbock erkannt.
Obwohl das CARB-Lager deutlich größeren axialen Versatz ermöglicht, waren dessen Wälzkörper einseitig stark verschlissen. Außerdem hatte das Pendelrollenlager seine axiale Position verändert, wobei dies bei einem Festlager nicht vorgesehen ist. Darüber hinaus wurde eine Exzentrizität der Welle festgestellt, wobei das Maß der Biegung bisher nur sporadisch ermittelt wurde. Das Ausmaß der durch Biegung und Längenausdehnung hervorgerufenen Verformung und die Auswirkungen auf die Lagerung konnten lediglich theoretisch untersucht werden, da diesbezüglich keine Messwerte vorliegen.

Die Ursachenbetrachtung wird durch die Variabilität des Schadensbildes, mögliche Montagefehler und den unbekannten zeitlichen Ablauf der Beschädigungen erschwert.

Da das Spektrum der Ursachen für die Überhitzung ebenso vielfältig ist wie deren Auftreten, gestaltet sich auch die Lösungsfindung kompliziert. Dennoch werden im Folgenden einige Maßnahmen, die zur Verbesserung der Situation beitragen können, beschrieben. 
Allgemein kommt bei Problemen durch zu hohe Temperatur eine Optimierung der Kühlung als Lösung in Frage, wenngleich die Temperaturmessung gezeigt hat, dass die Betriebstemperatur in der Regel in Ordnung ist. Dennoch kann den Überhitzungen durch eine erhöhte Wärmeabfuhr begegnet werden, wobei jedoch die metallurgischen Auswirkungen auf den Strang berücksichtigt werden müssen. Darüber hinaus muss geprüft werden, ob sich durch die Maßnahme der Wasserbedarf erhöht und ob dieser ggf. von den bestehenden Anlagen zur Wasserversorgung und -aufbereitung gedeckt werden kann.

Eine Möglichkeit besteht in der Anpassung der externen, also außerhalb der Rolleneinheit wirkenden Kühlanlage z. B. durch die Änderung von Art oder Anordnung der Düsen. Dies ist in Form des Nebeldüsenumbaus bereits geschehen, wobei der Effekt auf die Überhitzungen nur bedingt nachweisbar war. Bei der Optimierung der externen Kühlung sollte die Konzentration auf lokale Schwerpunkte erfolgen, um den Aufwand und die Auswirkungen auf den Strang möglichst gering zu halten.

Eine andere Möglichkeit ist die Verbesserung der internen Kühlung, also jener, die innerhalb der Rolleneinheit erfolgt. Diese Maßnahme wurde bereits in der Richtzone von Strang 1 durch die Verwendung mechanisch gekoppelter Rollenkörper mit integrierter Lagerkühlung umgesetzt.

Da das Klemmen der Rolle nach derzeitigem Stand nicht als Ursache für die Überhitzung in Frage kommt, wäre der Stillstand des Rollenkörpers durch mangelnden Kontakt zum Strang zu erklären. Um diesen zu gewährleisten, muss der Gießspalt an die sich ändernde Geometrie des Strangs angepasst werden. Damit ist insbesondere gemeint, dass sich die Maulweite mit steigender Stranglänge verringert, um auf die Schrumpfung infolge der Abkühlung des Stranges (soft reduction) zu reagieren. Die Maßnahme, den Gießspalt mit der vorhandenen Anlagentechnik zu korrigieren, war nicht erfolgreich, da weiterhin Überhitzungen auftraten. Es sollte also die Neusegmentierung der Anlage geprüft werden, wobei bereits ein Entwurf vorliegt, der jedoch überarbeitet und aktualisiert werden muss.
Da der konkrete Schadensverlauf und die auftretenden Wechselwirkungen noch nicht genau ermittelt werden konnten, sind weitere Untersuchungen notwendig, um die Ursache bzw. Ursachen der Überhitzungen sicher zu bestimmen. Dazu gehört auch die Vertiefung begonnener Analysen, wie der Dokumentation von Überhitzungsereignissen. Außerdem sollte der Zusammenhang zwischen Rotation und Überhitzung erneut und über einen längeren Zeitraum betrachtet werden. Dabei sind auch die Treiberrollen einzubeziehen, da auch hier per Thermovisionskamera Überhitzungen festgestellt wurden, obwohl diese jederzeit rotieren sollten.

Weiterhin sollten die Verformungen der Rolleneinheit sowie ihrer Komponenten, und auch des Segmentes genauer betrachtet werden. Dies gilt sowohl für die Deformation durch thermische als auch durch mechanische Belastung. Aufgrund der durchgeführten Temperaturmessung lagen zur Berechnung der Längenausdehnung relativ genaue und umfangreiche Daten vor. Dies ist in anderen Fällen, z. B. bei der Biegung der Achse, nicht der Fall, weshalb hier weitere Informationen als Grundlage für die Berechnungen notwendig sind. Zu diesem Zweck sollte die Verformung der relevanten Bauteile möglichst während des Gießprozesses ermittelt werden. Die Ergebnisse dieser Messungen müssen dann hinsichtlich der Ursachenfindung für die Überhitzung, z.B. durch fehlenden Kontakt zwischen Strang und Rollenkörper, ausgewertet werden. Auch die axiale Verschiebung des Rollenkörpers durch den Strang sollte untersucht werden. Wo die Durchführung solcher Messungen nicht unter vertretbarem Aufwand möglich ist, muss die Verformung auf andere Art bestimmt werden. Im Fall der Rollenkörper bietet bspw. Schwerdtfeger (1992: 402) eine gute Grundlage für die theoretische Betrachtung und Berechnung.

Außerdem muss, nachdem die Konzentration in dieser Arbeit auf das CARB-Lager gerichtet war, das Pendelrollenlager hinsichtlich seines Ausfalls genauer untersucht werden.

Weiterhin sollte der Anstieg der Rollenbelastung an der Sumpfspitze sowie in der Mitte des zweiten Segments der horizontalen Strangführung, welcher in Jänisch (2010) ermittelt wurde, als Ursache in Betracht gezogen werden. Hohe Aufmerksamkeit sollte zukünftig auch dem Zusammenbau der Rolleneinheiten gelten. Insbesondere wenn durch Montagefehler keine systematischen, sondern zufällige Abweichungen entstehen, ist deren Nachweis im späteren Verlauf kompliziert.

\section{LITERATUR}

ArcelorMittal (n/a) Zeichnungsarchiv ArcelorMittal Eisenhüttenstadt $\mathrm{GmbH}$.

Bernhard F (ed) (2014) Handbuch der Technischen Temperaturmessung, 2nd edn. VDI-Buch. Springer Vieweg, Berlin. ISBN: 978-3-642-24505-3. doi: 10.1007/978-3642-24506-0

Jänisch M (2010) Untersuchung der Belastungen von Strangführungsrollen im stationären Gießprozess. Master Thesis, Hochschule Lausitz

Rössel-Messtechnik (2012) Produktinformation 071: Mantel-Thermoelemente. Zur Temperaturmessung in Industrie, Labor und Forschung. http://www.roesselmesstechnik.de/webro-wAssets/docs/product-information/german/pi-071-metal-sheathed_de.pdf. Accessed 17 Aug 2015

Schwerdtfeger K (ed) (1992) Metallurgie des Stranggießens. Gießen und Erstarren von Stahl. Verlag Stahleisen, Düsseldorf. ISBN: 978-3-514-00350-7

\section{AUTOREN}

Jörn Bräske B. Eng

Prof. Dipl.-Ing. Thomas Mirre Technische Hochschule Wildau Maschinenbau

Dipl.-Ing. Steffen Klohs ArcelorMittal Eisenhüttenstadt Technologie Stahlwerk

E-Mail für Korrespondenz: joern.braeske@th-wildau.de 